\title{
CONTRACEPTIVE ACTION OF INTRA-UTERINE DEVICES IN THE RABBIT
}

\author{
J. H. MARSTON* AND M. G. CHANG \\ Worcester Foundation for Experimental Biology, \\ Shrewsbury, Massachusetts, U.S.A.
}

(Received 8th April 1968, revised 30th August 1968)

\begin{abstract}
Summary. A simple suture intra-uterine device (IUD), occupying almost the entire length of the uterine horn, was used to study the effect of an IUD upon conception in the rabbit.

After being mated, rabbits were killed at $84,132,156$ and $180 \mathrm{hr}$ and at 14 days. Additional animals were examined at 14 days after the IUD had been removed 84, 132 and $156 \mathrm{hr}$ after mating.

The IUD was not completely contraceptive, but it induced considerable pre-implantational embryonic death between 156 and $180 \mathrm{hr}$ after mating.
\end{abstract}

\section{INTRODUCTION}

Carleton \& Phelps (1933) were the first to investigate the contraceptive action of intra-uterine devices (IUDs) in rabbits. By using a variety of materials, they showed that IUDs usually exert an incomplete contraceptive action, so that implantation and foetal development could still occur. Conceptuses were more frequently found above than below the IUD, and it was suggested that mechanical obstruction of the uterine lumen might be one factor in the contraceptive action of IUDs in the rabbits.

Zipper, Delgado \& Guiloff (1963) found that the contraceptive effectiveness of IUD threads, which did not distend the uterus, was related to the length of the uterine lumen they occupied. Subsequent studies (Adams \& Eckstein, 1965a, b; Ledger, Virkar \& Irvin, 1966; Brown \& Foote, 1966; Dukelow, Perry \& Williams, 1967) also showed that the contraceptive effectiveness of IUDs in rabbits was variable; it usually increased as the IUD occupied a greater length of the uterine lumen, and was not related to the amount of uterine distension induced by the IUD.

These experiments all confirmed that implantation could occur in the presence of an IUD. The processes of ovulation and fertilization were not disturbed (Adams \& Eckstein, 1965a, b; Dukelow et al., 1967) but varying degrees of pre- and post-implantation embryonic death were induced by the IUDs. With the exception of Adams \& Eckstein (1965a, b), none of the studies attempted to define a point of action which might explain the ability of an IUD to cause preimplantational embryonic death in the rabbit.

* Present address: Physiological Laboratory, Cambridge CB2 3EG. 
This paper reports our experiments on the effect of a simple suture IUD, occupying almost the entire length of one uterine horn, upon embryonic development in the rabbit. Particular attention has been paid to development in the pre-implantation period, and some of our results have been noted in a previous communication (Marston \& Chang, 1964).

\section{MATERIALS AND METHODS}

Animals

Forty New Zealand White and thirteen cross-bred mature female rabbits were purchased from commercial breeders and maintained in the laboratory for 3 weeks before use.

\section{Insertion of an intra-uterine device}

Unilateral IUDs were inserted, with aseptic precautions, during mid-ventral laparotomy under pentobarbital/ether anaesthesia. They consisted of lengths of braided nylon suture thread (size 0) placed, at random, in one uterine horn so that they entered the uterine lumen about $5 \mathrm{~mm}$ below the utero-tubal junction and left about $5 \mathrm{~mm}$ above the cervix. The ends of the IUD thread pierced the anti-mesometrial uterine wall and were doubly knotted 1 to $2 \mathrm{~cm}$ away from the uterus to ensure that the IUD was not expelled. The rabbits were rested for at least 14 days after the operation.

A similar technique was employed to remove the IUDs after fertile mating.

\section{Mating and artificial insemination}

The rabbits were mated to males of proven fertility, or were artificially inseminated with a diluted ejaculate from these males. Immediately after mating or insemination they received an intravenous injection of $50 \mathrm{i}$.u. human chorionic gonadotrophin (HCG) to induce ovulation. Ovulation was assumed to occur 10 to $14 \mathrm{hr}$ after HCG injection (Harper, 1963). The day of ovulation was considered the 1st day of pregnancy or pseudopregnancy.

\section{Recovery of eggs and examination of tissues}

At autopsy 84 to $180 \mathrm{hr}$ after mating, the genital tract was completely removed and the Fallopian tubes, uterine horns and vagina were then individually flushed with Ringer's solution. Eggs were recovered and examined according to Chang (1952). Blastocysts recovered at $156 \mathrm{hr}$ after mating were examined according to Moog \& Lutwak Mann (1958).

The number of ovulation spots or corpora lutea on each ovary was determined by gross examination. The uterine horns were fixed in aqueous Bouin's solution or $10 \%$ buffered neutral formol. Transverse sections were cut at 7 to $10 \mu$ and stained routinely with haematoxylin and eosin, by the PAS technique, and by the oil red $\mathrm{O}$ technique. The uterine horns of rabbits killed $180 \mathrm{hr}$ after mating were first examined by trans-illumination which demonstrated the presence of implantation chambers as areas of greater transparency: their position was recorded. 
Attempts to induce a decidual reaction

Four does fitted with unilateral IUDs were subjected to uterine traumatization at 132 and $156 \mathrm{hr}$ after the injection of HCG (two does in each group), by fitting both uterine horns with a traumatizing thread in the same way as for an IUD thread. They were killed on the 14th day of pseudopregnancy.

\section{Terminology}

The ovary, Fallopian tube or uterine horn from the side fitted with an IUD will be identified by the prefix 'IUD'; the contralateral organs will be prefixed by 'control'.

\section{Ovulation}

\section{RESULTS}

Forty does were used in the main series of experiments. The mean number of corpora lutea or ovulation spots on their control and IUD ovaries was $5 \cdot 6 \pm 0.3$ (standard error) and $5 \cdot 6 \pm 0 \cdot 4$, respectively. Thus, the IUD used in the present study had no effect on the ovulation rate.

\section{Fertilization}

Table 1 presents the observations on the recovery of eggs and embryos from the uterine horns of animals killed 84 to $180 \mathrm{hr}$ after mating.

The IUD had no obvious effect upon fertilization and, by inference, capacitation and the transport of spermatozoa through the genital tract in the presence of an IUD was adequate for the maintenance of normal fertility.

\section{Transport of eggs through the genital tract}

Eggs were recovered from the uterine horns $84 \mathrm{hr}$ after mating, and none was present in the Fallopian tubes. Two animals each yielded a degenerate blastocyst from the vaginal lavage 84 and $132 \mathrm{hr}$, respectively, after mating. However, degenerate embryos were not recovered from the vagina at later time intervals, and there was no indication of loss of embryos from the IUD uterine horn at 156 and $180 \mathrm{hr}$. There was no evidence of abnormal transport of eggs through the genital tract in the presence of an IUD.

\section{Embryonic development}

Table 1 indicates that embryonic development was normal in the presence of an IUD at 84, 132 and $156 \mathrm{hr}$ after mating.

At $84 \mathrm{hr}$ the eggs were at the early blastocyst stage. At $132 \mathrm{hr}$ expanded blastocysts were recovered; twenty-one from the control uterine horn had a mean diameter (two axes) of $1.76 \pm 0.10 \mathrm{~mm}$, while twenty-two from the IUD horn measured $1 \cdot 67 \pm 0 \cdot 10 \mathrm{~mm}$. The differences in blastocyst diameter were not significant.

At $156 \mathrm{hr}$ after mating, blastocysts were classified as normal if they were fully expanded to a total diameter of more than $1.0 \mathrm{~mm}$. Of nineteen blastocysts recovered from the control uterine horns, seventeen were normal with a mean diameter of $4 \cdot 12 \pm 0.20 \mathrm{~mm}$; the remaining eggs were expanded blastocysts but measured only $1.0 \mathrm{~mm}$ in diameter. Fifteen normal blastocysts with a mean 
diameter of $3.83 \pm 0.31 \mathrm{~mm}$ were recovered from the IUD uterine horns, together with one small expanded blastocyst $(1.0 \mathrm{~mm}$ diameter) and two collapsed blastocysts which had probably been expanded. Seven normal blastocysts from the IUD horns of two rabbits started to collapse soon after recovery, and were completely collapsed at the time of fixation. After staining, the mean diameters (two axes) of the germ discs of fifteen control and nine IUD blastocysts were, respectively, $0.96 \pm 0.04 \mathrm{~mm}$ and $0.87 \pm 0 \cdot 10 \mathrm{~mm}$. There were no significant differences in the dimensions of normal blastocysts recovered from the control and IUD uterine horns $156 \mathrm{hr}$ after mating.

\section{Implantation}

In the control uterine horns of rabbits killed at $180 \mathrm{hr}, 90 \%$ of the ovulated eggs were accounted for by the presence of implantation chambers evenly spaced along the horn (Table 1). In the IUD horns, a total of nine implantation chambers was found in three animals (Pl. 1, Figs. 6 and 7). One IUD horn showed six implantation chambers, one of which was degenerating, evenly spaced along its length and in complete contact with the IUD thread. This horn was not flushed, although the ipsilateral ovary had nine corpora lutea. The second IUD horn had a single implantation chamber close to its mid-point in complete contact with the IUD thread: five degenerating blastocysts were flushed from the horn to tally with six corpora lutea on the IUD ovary. Five degenerating blastocysts (Pl. 1, Fig. 1) were flushed from the third IUD horn, which measured $11 \mathrm{~cm}$ in length and contained two implantation chambers in contact with the IUD thread at a distance of 3 and $6 \mathrm{~cm}$ from the uterotubal junction: seven corpora lutea were found on the IUD ovary. The fourth animal had five corpora lutea on the IUD ovary and five degenerating blastocysts were flushed from the IUD uterine horn. The fifth animal had no implantation chambers in the IUD uterine horn, which was not flushed, and six corpora lutea were counted on the IUD ovary.

All fifteen degenerating blastocysts flushed from the IUD horns were estimated to have been expanded to a size equal to or larger than that of the blastocysts recovered $156 \mathrm{hr}$ after mating. Thirteen blastocysts had collapsed within the zona pellucida, and two were naked.

\section{Foetal development}

On the 14th day of pregnancy (Table 2) foetal development in the control uterine horn accounted for $80 \%$ of the ovulated eggs. In the IUD horn, there

\section{EXPLANATION OF PLATE 1}

All sections at $10 \mu$ and stained with haematoxylin-eosin, except for those in Figs. 8 and 9 which were stained by the PAS technique. $x$, Position of IUD in Figs. 3, 5 and 7.

Frg. 1. Degenerate blastocysts from IUD uterine horn at $180 \mathrm{hr}$ after mating. $\times$ about 10.

Figs. 2 and 3. Control and IUD uterine horns at $132 \mathrm{hr}$ after mating. $\times 5$.

Figs. 4 and 5 . Control and IUD uterine horns at $156 \mathrm{hr}$ after mating. $\times 5$.

Fics. 6 and 7. Implantations in control and IUD uterine horns at $180 \mathrm{hr}$ after mating. $\times 5$.

FIGs. 8 and 9. Uterine traumatization-control and IUD uterine horns on the 14th day of pseudopregnancy. $\times 5$. 

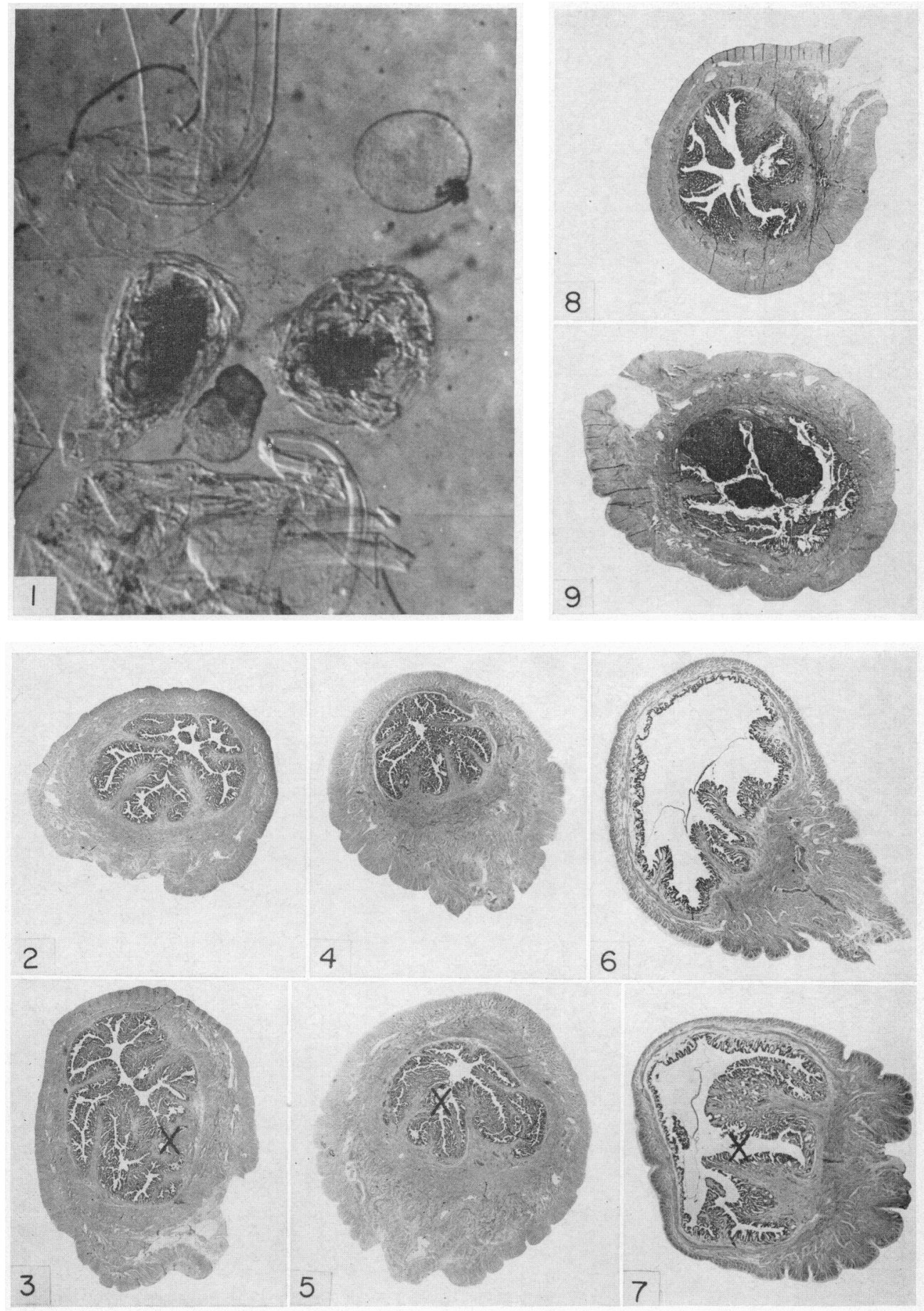

(Facing 1. 412) 


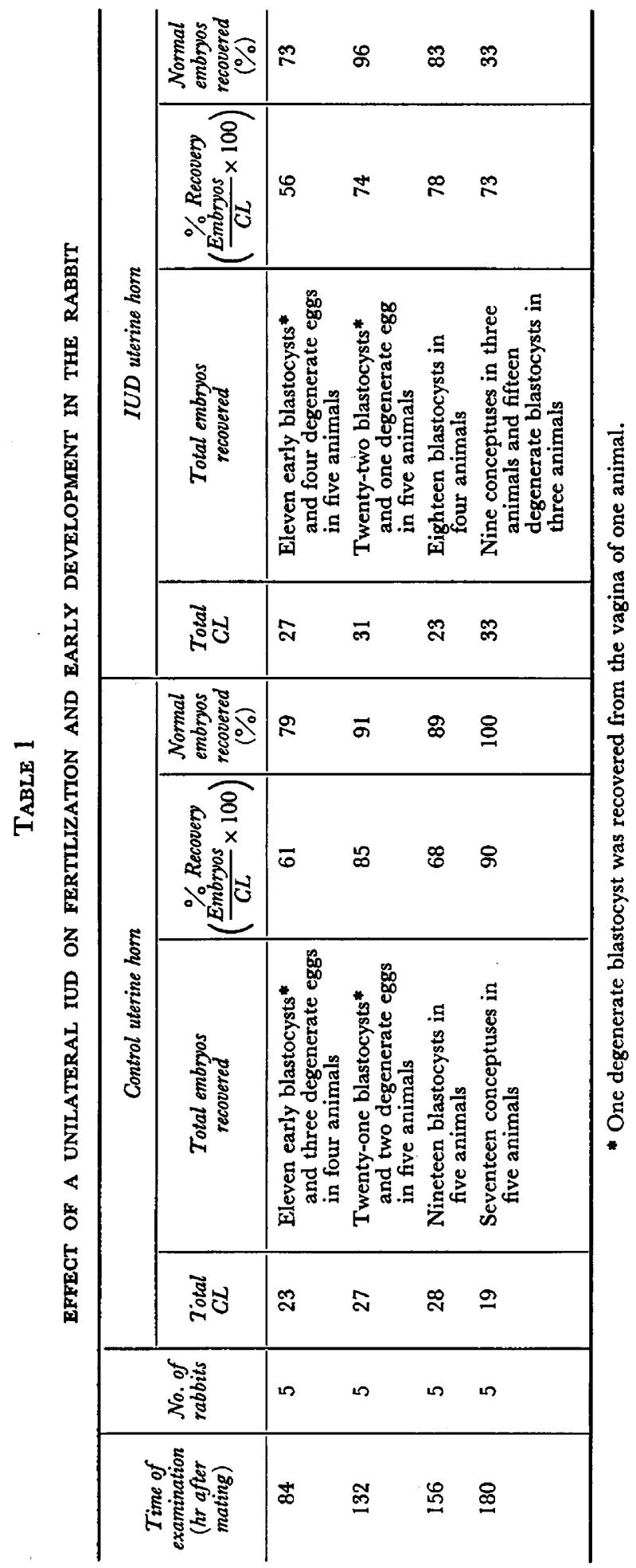




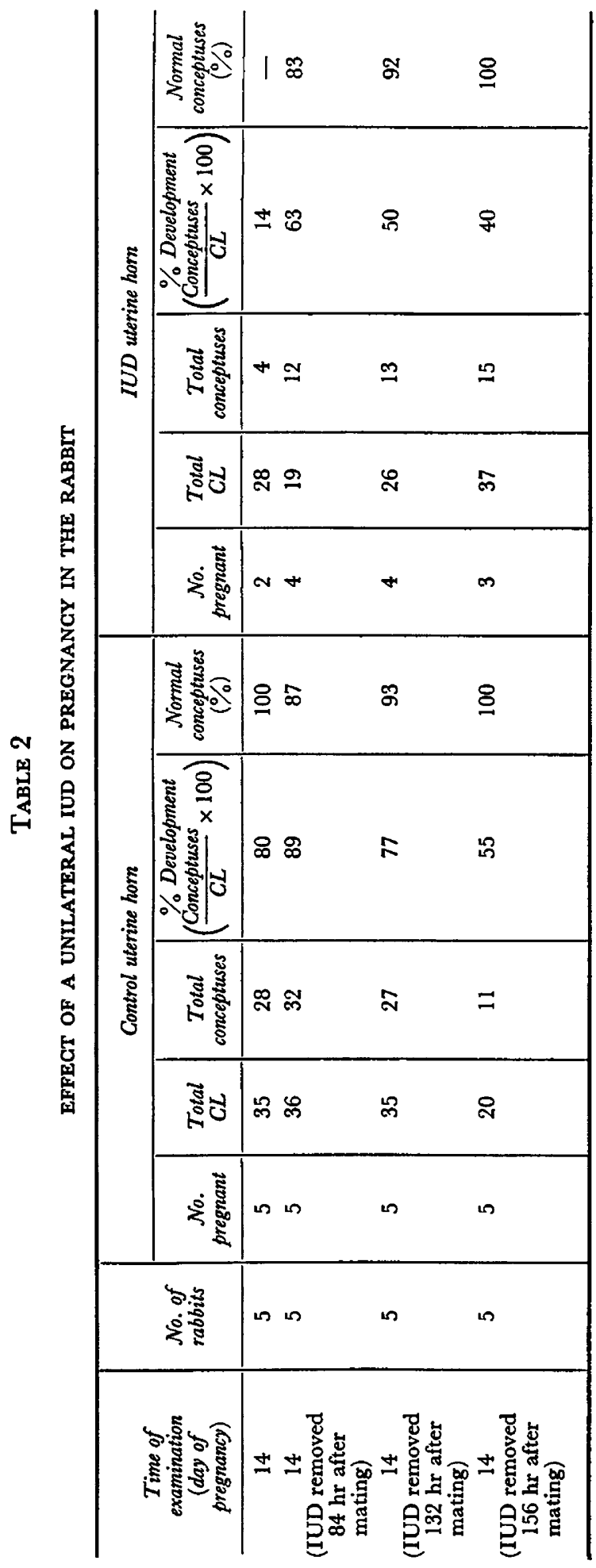


were no normal foetuses, and two animals yielded a total of four degenerate conceptuses. The degenerate conceptuses were completely in contact with the IUD thread which passed across the implantation chamber.

Removal of the IUD thread 84 to $156 \mathrm{hr}$ after mating allowed normal foetal development to occur (Table 2). Not all the animals were pregnant in the IUD uterine horn, but the percentage development in the pregnant IUD horns $(57$ to $75 \%$ ) was close to that in the control horns ( 55 to $89 \%$ ).

\section{Histology of the uterus in the presence of an intra-uterine device}

The IUD uterine horns did not show any gross abnormality of endometrial or myometrial histology (Pl. 1, Figs. 2 to 5 ) but a constant lesion was closely associated with the IUD thread. It consisted of superficial erosion of the endometrium with loss of the epithelium, and a greater or lesser degree of leucocytic infiltration into the endometrial stroma. The IUD thread occupied a variable position in the uterine lumen, but it usually lay between or immediately lateral to the two large mesometrial (placental) folds of endometrium. Leucocytic infiltration of the thread itself seldom occurred, and when it did, was of trivial significance.

The non-conceptual areas of the IUD and control uterine horns did not show gross differences at $180 \mathrm{hr}$ after mating although the IUD thread had caused local endometrial erosion and slight leucocytic infiltration. In areas where implantation was occurring, the anti-mesometrial attachment of trophoblast to the endometrium could be identified, even in the presence of the IUD thread. Embryonic structures also appeared to be normal in the presence of the IUD thread (Pl. 1, Fig. 7).

The uterine flushes recovered from the control and IUD uterine horns at 84 to $180 \mathrm{hr}$ contained a variable amount of cellular and leucocytic debris.

\section{Attempts to induce a decidual reaction}

The control uterine horns did not show an obvious reaction to traumatization apart from localized erosion of the endometrium in contact with the traumatizing thread. The morphology of the uterine horns was similar to that observed on the 14th day in untreated pseudopregnant does, or in the non-conceptual areas of pregnant does.

By contrast, all the IUD uterine horns showed hypertrophy of the mesometrial (placental) folds of endometrium. This was caused by hypertrophy and hyperplasia of the endometrial stroma. The stromal cells showed an accumulation of lipid, and an intense PAS reaction, which was abolished following diastase treatment (Pl. 1, Figs. 8 and 9).

\section{DISCUSSION}

There is general agreement that implantation in the rabbit, as judged by the first antimesometrial attachment between the trophoblast and the endometrium, occurs between 156 and $180 \mathrm{hr}$ after mating. We do not know exactly when implantation occurred in our rabbits, but our results show that nearly two-thirds of the blastocysts developing normally within the IUD uterine horn 
at $156 \mathrm{hr}$ had degenerated by $180 \mathrm{hr}$ after mating. The appearance of the degenerate blastocysts suggested that they had not implanted. Thus, in our experiments, the most significant effect of a thread-IUD occupying almost the entire length of the uterine lumen was to cause pre-implantational embryonic death, between 156 and $180 \mathrm{hr}$ after mating.

Adams \& Eckstein (1965a, b) also concluded that an IUD exerted some form of local inhibitory action on the unimplanted embryo at $144 \mathrm{hr}$ after mating. Blastocysts from uteri fitted with their less effective type of IUD thread were said to be significantly smaller in total area and disc area than those from control horns. This effect was apparently not observed in uteri fitted with the more effective type of IUD thread. We have not observed any significant differences in the dimensions of control and IUD blastocysts at either 132 or $156 \mathrm{hr}$ after mating.

The fact that we could recover degenerate blastocysts from the IUD uteri suggests that the IUD had not induced gross changes in the pattern of uterine motility. This does not, however, rule out the possibility that an IUD prevents implantation by causing more subtle, local changes in uterine motility. The mechanical presence of an IUD at the site of implantation could also upset surface to surface attachment of the trophoblast to the endometrial epithelium. These effects would presumably be exerted at the time of blastocyst attachment, and one might expect that the blastocysts would have, by then, been freed from their zonae pellucidae. In fact, the degenerate blastocysts were usually recovered with their zonae intact.

In the rabbit uterus, an IUD may cause some more or less specific change in the uterine milieu such that it becomes toxic to blastocysts during the critical pre-implantation period. Our results show that blastocysts were able to develop normally in the IUD uterus for at least 3 days; during this period, they were free within the uterine lumen and nourished by secretions from the whole of the endometrial epithelium. However, implantation involves a strictly localized interaction between the endometrial epithelium and the blastocyst. The presence of an IUD thread may so disturb the pattern of endometrial and/ or blastocyst metabolism at the implantation site as to cause the death of most of the blastocysts.

In the rabbit, an IUD appears to cause local disturbances in uterine function. Our results agree with previous reports that an IUD does not completely prevent implantation. This partial effectiveness contrasts with the complete contraceptive action of IUDs in other species (cf. Marston \& Kelly, 1966). It may be related to particular features of the rabbit's central and superficial implantation. In our experiments, however, the IUD threads may not have been completely contraceptive because they tended to lie between the placental endometrial folds, and in this position their effects may have been limited. More rigid types of IUD (Ledger et al., 1966) and free-ended threads (Adams \& Eckstein, 1965b) exert a greater contraceptive effect in the rabbit. This may be because they do not become embedded in the endometrium.

The IUDs used by other workers (Adams \& Eckstein, 1965a, b; Brown \& Foote, 1966; Dukelow et al., 1967) appear to have usually caused only a small or variable amount of pre-implantational embryonic death, but post-implanta- 
tional death was more extensive. Once implantation has occurred in an IUD uterus, then the gross presence of a foreign body could reasonably be expected to disturb the balance of function within the uterus and cause death of the embryo. Adams \& Eckstein (1965a) have also suggested that the post-implantational embryonic death was the result of interference with placental function. The apparent action of an IUD in causing post-implantational embryonic death in the rabbit may not be relevant to the basic problems of IUD action in women and experimental animals.

The published studies show that an IUD has no effect on the ovulation rate of the IUD ovary and both the control and IUD corpora lutea appear to undergo normal development. Implantation occurs normally in the control uterine horn and gestation can be completed. Thus, the hormonal background to pregnancy in the rabbit is not affected by an IUD. Dukelow et al. (1967) reported a high incidence of embryonic death in the control horn during the second half of pregnancy. Their evidence was not extensive and the increased mortality was not statistically significant. Adams \& Eckstein (1965a) found that the embryonic death occurring in the control horn was similar to that expected for normal pregnancies.

At present, there is little information to suggest how an IUD induces preimplantational embryonic death in the rabbit. Like Carleton \& Phelps (1933), Zipper et al. (1963) and Adams \& Eckstein (1965a), we have observed localized areas of endometrial erosion and varying amounts of leucocytic infiltration in close association with the IUD. These changes resembled those of pressure atrophy. Similar observations were made by Kar \& Kamboj (1965) in oestrous rabbits, Ledger \& Bickley (1966) in recently ovulated rabbits and Parr, Schaedler \& Hirsch (1967) in does on the 14th day of pregnancy.

The available biochemical data have usually been obtained from oestrous rabbits (Carleton \& Phelps, 1933; Kar, Kamboj, Chowdhury \& Chowdhury, 1965; Dukelow et al., 1967). In the two latter studies, the gross and probably unphysiological procedures of tubal ligation and uterine ligation were used to obtain long-term, accumulated specimens of tubal and uterine fluid. Studies on pseudopregnant IUD uteri have yielded no relevant information (Zipper et al., 1963; Dukelow et al., 1967; Parr et al., 1967).

\section{ACKNOWLEDGMENTS}

This work was initiated with a grant from the Population Council and continued with the help of a grant from the Ford Foundation to Dr P. Eckstein. Dr W. A. Kelly has given helpful advice in preparing this manuscript.

\section{REFERENCES}

Adams, C. E. \& Eckstein, P. (1965a) The effect of intrauterine foreign bodies on pregnancy in the rabbit. Fert. Steril. 16, 508.

Adams, C. E. \& Eckstein, P. (1965b) Effects of intrauterine silk threads on location and survival of conceptuses in the rabbit. F. Reprod. Fert. 9, 351.

Brown, E. \& FoOTE, R. H. (1966) The effect of plastic devices in the uterine lumen on pregnancy and parturition in the rabbit. F. Reprod. Fert. 12, 373.

Carleton, H. M. \& Phelps, H. J. (1933) Experimental observations on the Gräfenberg ring contraceptive methods. 7. Obstet. Gynaec. Br. Commonw. 40, 81 . 
Chang, M. C. (1952) Fertilizability of rabbit ova and the effects of temperature in vitro on their subsequent fertilization and activation in vivo. F. exp. Zool. 121, 351.

Dukelow, W. R., Perry, H. A. \& Williams, W. L. (1967) Intrauterine device-induced embryonic mortality in rabbits. Fert. Steril. 18, 557.

HARPER, M. J. K. (1963) Ovulation in the rabbit: the time of follicular rupture and expulsion of the eggs in relation to injection of luteinizing hormone. F. Endocr. 26, 307.

KAR, A. B. \& KAMBOJ, V. P. (1965) Effect of intrauterine contraceptive devices on histological and histochemical changes in rabbit uterus. Indian F. exp. Biol. 3, 141.

Kar, A. B., KamboJ, V. P., Chowdhury, S. R. \& Chowdhury, A. R. (1965) Effect of a contraceptive suture on biochemical composition of the rabbit Fallopian tube fluid. Indian f. exp. Biol. 3, 268.

LEDGER, W.J. \& BickLeY, J. E. (1966) Effect of a plastic foreign body on the genital tract of the female rabbit. Obstet. Gynec., N.Y. 27, 658.

Ledger, W. J., Virkar, K. D. \& Irvin, L. A. (1966) Effects of a plastic intrauterine device upon implantation in the rabbit. Obstet. Gynec., $\mathcal{N} . Y .28,521$.

Marston, J. H. \& Chang, M. C. (1964) Action of intrauterine foreign bodies in the rat and rabbit. Proc. 2nd int. Conf. on Intra-uterine Contraception, Excerpta Med. Int. Congr. Ser. 86, 243.

Marston, J. H. \& Kelly, W. A. (1966) The effects and mode of action of intrauterine devices. Vet. Rec. 79, 644 .

Moog, F. \& LuTwak-MANn, C. (1958) Observations on rabbit blastocysts prepared as flat mounts. 7. Embryol. exp. Morph. 6, 67.

PARr, E. L., Schaedler, R. W. \& Hirsch, J. G. (1967) The relationship of polymorphonuclear leukocytes to infertility in uteri containing foreign bodies. J. exp. Med. 126, 523.

Zipper, J., Delgado, R. \& Guiloff, E. (1963) Estudios experimentales del mechanismo de accion de cuerpos intra-uterinos. Rev. chil. Obstet. Ginec. 28, 18. 